\title{
Dissipative Quantum Church-Turing Theorem
}

\author{
M. Kliesch, ${ }^{1,2}$ T. Barthel, ${ }^{1,2}$ C. Gogolin, ${ }^{1,2}$ M. Kastoryano, ${ }^{3}$ and J. Eisert ${ }^{1,2}$ \\ ${ }^{1}$ Dahlem Center for Complex Quantum Systems, Freie Universität Berlin, 14195 Berlin, Germany \\ ${ }^{2}$ Institute for Physics and Astronomy, University of Potsdam, 14476 Potsdam, Germany \\ ${ }^{3}$ Niels Bohr Institute, University of Copenhagen, 2100 Copenhagen, Denmark
}

(Received 10 June 2011; published 12 September 2011)

\begin{abstract}
We show that the time evolution of an open quantum system, described by a possibly time dependent Liouvillian, can be simulated by a unitary quantum circuit of a size scaling polynomially in the simulation time and the size of the system. An immediate consequence is that dissipative quantum computing is no more powerful than the unitary circuit model. Our result can be seen as a dissipative Church-Turing theorem, since it implies that under natural assumptions, such as weak coupling to an environment, the dynamics of an open quantum system can be simulated efficiently on a quantum computer. Formally, we introduce a Trotter decomposition for Liouvillian dynamics and give explicit error bounds. This constitutes a practical tool for numerical simulations, e.g., using matrix-product operators. We also demonstrate that most quantum states cannot be prepared efficiently.
\end{abstract}

DOI: 10.1103/PhysRevLett.107.120501

PACS numbers: 03.67.Ac, 02.60.Cb, 03.65.Yz, 89.70.Eg

One of the cornerstones of theoretical computer science is the Church-Turing thesis [1,2]. In its strong formulation it can be captured in the following way [3,4]: "A probabilistic Turing machine can efficiently simulate any realistic model of computation." As such, it reduces any physical process - that can intuitively be thought of as a computational task in a wider sense- to what an elementary standard computer can do. Needless to say, in its strong formulation, the Church-Turing thesis is challenged by the very idea of a quantum computer, and hence by a fundamental physical theory that initially was thought to be irrelevant for studies of complexity. There are problems a quantum computer could efficiently solve that are believed to be intractable on any classical computer.

In this way, it seems that the strong Church-Turing thesis has to be replaced by a quantum version [2]. Colloquially speaking, the quantum Church-Turing thesis says that any process that can happen in nature that one could think of as being some sort of computation is efficiently simulatable.

Strong quantum Church-Turing thesis: Every quantum mechanical computational process can be simulated efficiently in the unitary circuit model of quantum computation.

Indeed, this notion of quantum computers being devices that can efficiently simulate natural quantum processes, being known under the name "quantum simulation," is the topic of an entire research field initiated by the work of Feynman [5]. Steps towards a rigorous formulation have been taken by Lloyd [6] and many others [7].

Quite surprisingly, a very important class of physical processes appears to have been omitted in the quest for finding a sound theory of quantum simulation, namely, dissipative quantum processes. Such processes are particularly relevant since, in the end, every physical process is to some extent dissipative. If one aims at simulating a quantum process occurring in a lab, one cannot, however, reasonably require the inclusion of all modes of the environment to which the system is coupled into the simulation. Otherwise, one would always have to simulate all the modes of the environment, eventually of the entire Universe, rendering the task of simulation obsolete and futile. We argue that the most general setting in which one can hope for efficient simulatability is the one of Markovian dynamics [8] with arbitrary piecewise continuous time dependent control [9]. In any naturally occurring process the Liouvillian $\mathcal{L}$ determining the equation of motion

$$
\frac{d}{d t} \rho(t)=\mathcal{L}_{t}(\rho(t))
$$

of the system state $\rho$ is $k$-local. This means that the system is multipartite and $\mathcal{L}$ can be written as a sum of Liouvillians each acting nontrivially on at most $k$ subsystems. In fact, all natural interactions are two-local in this sense. Since we are interested in processes which can be viewed as a computation, we assume that the subsystems are of fixed finite dimension. This is arguably the broadest class of natural physical processes that should be taken into account in a dissipative Church-Turing theorem and includes the Hamiltonian dynamics of closed systems as a special case.

In this work, we show the following.

(i) Every time evolution generated by a $k$-local time dependent Liouvillian can be simulated by a unitary quantum circuit with resources scaling polynomially in the system size $N$ and simulation time $\tau$.

(ii) As a corollary, we obtain that the dissipative model for quantum computing [11] can be reduced to the circuit model-proving a conjecture that was still open. 
(iii) Technically, we show that the dynamics can be approximated by a Trotter decomposition, giving rise to a circuit of local channels, actually being reminiscent of the situation of unitary dynamics. In particular, in order to reach a final state that is only $\epsilon$ distinguishable from the exactly time evolved state, it will turn out to be sufficient to apply a circuit of $\mathrm{Km}$ local quantum channels, where

$$
m=\left\lceil\max \left(\frac{2 c K^{2} \tau^{2}}{\epsilon}, \frac{\tau b}{\ln 2}\right)\right\rceil
$$

is the number of time steps, $K \leq N^{k}$ is the number of local terms in the Liouvillian, and $b$ and $c$ are constants independent of $N, \tau, K$, and $\epsilon$. Some obstacles of naive attempts to simulate dissipative dynamics are highlighted, and the specific role of the appropriate choice of norms is emphasized.

(iv) We also show that most quantum states cannot be prepared efficiently.

(v) In addition, the Trotter decomposition with our rigorous error bound is a practical tool for the numerical simulation of dissipative quantum dynamics on classical computers.

Setting.-We consider general quantum systems consisting of $N$ subsystems of Hilbert-space dimension $d$. The dynamics is described by a quantum master equation (1) with a $k$-local Liouvillian of the form

$$
\mathcal{L}=\sum_{\Lambda \subset[N]} \mathcal{L}_{\Lambda},
$$

where $[N]:=\{1,2, \ldots, N\}$ and $\mathcal{L}_{\Lambda}$ are strictly $k$-local Liouvillians. The subscript $\Lambda$ means that the respective operator or superoperator acts nontrivially only on the subsystem $\Lambda$ and we call an operator or superoperator strictly $k$-local if it acts nontrivially only on at most $k$ subsystems. Each of the Liouvillians $\mathcal{L}_{\Lambda}$ can be written [10] in Lindblad form [12]

$$
\mathcal{L}_{\Lambda}=-i\left[H_{\Lambda}, \cdot\right]+\sum_{\mu=1}^{d^{k}} \mathcal{D}\left[L_{\Lambda, \mu}\right],
$$

where $\mathcal{D}[X](\rho):=2 X \rho X^{\dagger}-\left\{X^{\dagger} X, \rho\right\}$ and may depend on time piecewise continuously. In particular, we do not require any bound on the rate at which the Liouvillians may change.

The propagators $T_{\mathcal{L}}(t, s)$ are the family of superoperators defined by

$$
\rho(t)=T_{\mathcal{L}}(t, s)(\rho(s))
$$

for all $t \geq s$. They are completely positive and trace preserving (CPT) and uniquely solve the initial value problem

$$
\frac{d}{d t} T(t, s)=\mathcal{L}_{t} T(t, s), \quad T(s, s)=\mathrm{id},
$$

where id denotes the identity map.
The main result, which is a bound on the error of the Trotter decomposition, will be somewhat reminiscent of the Trotter formula for time dependent Hamiltonian dynamics derived in Ref. [13]. The main challenge comes from the fact that we are dealing with superoperators rather than operators. The key to a meaningful Trotter decomposition is the choice of suitable norms for these superoperators. The physically motivated and strongest norm is the one arising from the operational distinguishability of two quantum states $\rho$ and $\sigma$, which is given by the trace distance $\operatorname{dist}(\rho, \sigma):=\sup _{0 \leq A \leq \mathbb{1}} \operatorname{tr}[A(\rho-\sigma)]$. The trace distance coincides up to a factor of $1 / 2$ with the distance induced by the Schatten 1-norm $\|\cdot\|_{1}$, where the Schatten $p$-norm of a matrix $A$ is $\|A\|_{p}:=\left[\operatorname{tr}\left(|A|^{p}\right)\right]^{1 / p}$. Therefore, we measure errors of approximations of superoperators with the induced operator norm, which is the so-called $(1 \rightarrow 1)$-norm. In general the $(p \rightarrow q)$-norm of a superoperator $T \in \mathcal{B}(\mathcal{B}(\mathcal{H}))$ is defined as [14]

$$
\|T\|_{p \rightarrow q}:=\sup _{\|A\|_{p}=1}\|T(A)\|_{q} .
$$

The difficulty in dealing with these norms lies in the fact that for $p<\infty$ the $p$-norm does not respect $k$-locality, e.g., $\left\|A \otimes \mathbb{1}_{n \times n}\right\|_{1}=n\|A\|_{1}$. This problem is overcome by using the Lindblad form of the strictly $k$-local Liouvillians. In the end, all bounds can be stated in terms of the largest operator norm $\left\|X_{t}\right\|_{\infty}$ of the Lindblad operators $X \in \mathcal{L}_{\Lambda}$ of the strictly $k$-local terms. The notation $X \in \mathcal{L}_{\Lambda}$ means that $X$ is one of the operators occurring in the Lindblad representation (4) of $\mathcal{L}_{\Lambda}$. From now on we assume that this largest operator norm $a$ is everywhere bounded by a constant of order 1 and, in particular, independent of $N$, i.e., $a \in O(1)$.

Main result.-One can always approximate any dissipative dynamics generated by a $k$-local Liouvillian acting on $N$ subsystems, even allowing for piecewise continuous time dependence, by a suitable Trotter decomposition. The error made in such a decomposition can be bounded rigorously.

Theorem 1 (Trotter decomposition of Liouvillian dynamics). Let $\mathcal{L}=\sum_{\Lambda \subset[N]} \mathcal{L}_{\Lambda}$ be a $k$-local Liouvillian that acts on $N$ subsystems with local Hilbert-space dimension $d$. Furthermore, let $\mathcal{L}$ be piecewise continuous in time with the property that $a=\max _{\Lambda} \max _{X \in \mathcal{L}_{\Lambda}} \sup _{t \geq 0}\left\|X_{t}\right\|_{\infty}$ $\in O(1)$. Then the error of the Trotter decomposition of a time evolution up to time $\tau$ into $m$ time steps is

$$
\left\|T_{\mathcal{L}}(\tau, 0)-\prod_{j=1}^{m} \prod_{\Lambda \subset[N]} T_{\mathcal{L}_{\Lambda}}\left(\tau \frac{j}{m}, \tau \frac{j-1}{m}\right)\right\|_{1 \rightarrow 1} \leq \frac{c K^{2} \tau^{2} e^{b \tau / m}}{m},
$$

where $c \in O\left(d^{2 k}\right), \quad b \in O\left(d^{k}\right)$, and $K \leq N^{k}$ is the number of strictly $k$-local terms $\mathcal{L}_{\Lambda} \neq 0 . T_{\mathcal{L}_{\Lambda}}\left(\tau \frac{j}{m}, \tau \frac{j-1}{m}\right)$ can be replaced by the propagator $T_{\mathcal{L}_{\Lambda}}$ av $\left(\tau \frac{j}{m}, \tau \frac{j-1}{m}\right)=$ $\exp \left(\tau \mathcal{L}_{\Lambda}^{\mathrm{av}} / m\right)$ of the average Liouvillian 


$$
\mathcal{L}_{\Lambda}^{\mathrm{av}}=\frac{m}{\tau} \int_{\tau(j-1) / m}^{\tau j / m} \mathcal{L}_{\Lambda} d t
$$

without changing the scaling (8) of the error.

All constants are calculated explicitly in the Supplemental Material [15]. The supremum in $a$ can be replaced by suitable time averages over the time steps such that $\left\|X_{t}\right\|_{\infty}$ can be large for small times. Before we turn to the proof of this result, we discuss important implications.

Implication 1 (Dissipative Church-Turing theorem). Time dependent Liouvillian dynamics can be simulated efficiently in the standard unitary circuit model.

Using the Stinespring dilation [16], each of the $\mathrm{Km}$ propagators $T_{\mathcal{L}_{\Lambda}}\left(\tau \frac{j}{m}, \tau \frac{j-1}{m}\right)$ can be implemented as a unitary $U_{\Lambda}^{j}$ acting on the subsystem $\Lambda$ and an ancilla system of size at most $d^{2 k}$. These unitaries can be decomposed further into circuits $\tilde{U}_{\Lambda}^{j}$ of at most $n=O\left(\log ^{\alpha}\left(1 / \epsilon_{\mathrm{SK}}\right)\right)$ gates from a suitable gate set using the Solovay-Kitaev algorithm [17] with $\alpha<4$ such that $\left\|U_{\Lambda}^{j}-\tilde{U}_{\Lambda}^{j}\right\|_{\infty} \leq \epsilon_{\mathrm{SK}}$. Note that for pure states, we have $\frac{1}{2} \| U|\psi\rangle\left\langle\psi\left|U^{\dagger}-\tilde{U}\right| \psi\right\rangle \times$ $\langle\psi| \tilde{U}^{\dagger}\left\|_{1} \leq\right\| U-\tilde{U} \|_{\infty} \leq \epsilon_{\mathrm{SK}}$ and the 1-norm is nonincreasing under partial trace. The full error is bounded by the error from the Trotter approximation (8) plus the one arising from the Solovay-Kitaev decomposition, in $(1 \rightarrow 1)$-norm bounded by $K m \epsilon_{\mathrm{SK}}$.

At this point a remark on the appropriate degree of generality of the above result is in order. The proven result applies to dynamics under arbitrary piecewise continuous time dependent $k$-local Liouvillians. It does not include non-Markovian dynamics as often resulting from strong couplings. However, not only this result, but no dissipative Church-Turing theorem, can or should cover such a situation: Including highly non-Markovian dynamics would mean to also include extreme cases such as an evolution implementing a swap gate that could write the result of an incredibly complicated process happening in the huge environment into the system. In such an intertwined situation it makes only limited sense to speak of the time evolution of the system alone in the first place. On the other hand, in practical simulations of non-Markovian dynamics, where the influence of memory effects is known, pseudomodes can be included [18], thereby rendering the above results again applicable.

It has been shown recently [19] that the set of states that can be reached from a fixed pure reference state by $k$-local, time dependent Hamiltonian dynamics is exponentially smaller than the set of all pure quantum states. In fact, a more general statement holds true (see Supplemental Material [15]).

Implication 2 (Limitations of efficient state generation). Let $\mathcal{S}_{\tau}^{\rho}$ be the set of states resulting from the time evolution of an arbitrary initial state $\rho$ under all possible (time dependent) $k$-local Liouvillians up to some time $\tau$. For times $\tau$ that are polynomial in the system size, the relative volume of $\mathcal{S}_{\tau}^{\rho}$ (measured in the operational metric induced by the 1-norm) is exponentially small.

Finally, Theorem 1 also provides a rigorous error bound for the simulation of local time dependent Liouvillian dynamics on a classical computer. Even though classical simulation of quantum mechanical time evolution is generally believed to be hard in time, we have the following result.

Implication 3 (Simulation on classical computers). For fixed simulation time and efficiently evaluable initial states [20], dissipative dynamics can be simulated efficiently in the system size on classical computers, e.g., using a variant of time-dependent density matrix renormalization group.

This establishes a mathematically sound foundation for simulation techniques based on Trotter decomposition that have previously been used without proving that the approximation is actually possible; see, e.g., Ref. [21]. Recently, CPT maps like the local channels in the Trotter decomposition (8) have even been implemented in the lab [22].

Proof of theorem 1.-We now turn to the proof of the main result. First we will find $(1 \rightarrow 1)$-norm estimates (i) for $T$ and (ii) for $T^{-}$which will be used frequently. In the next step (iii) we derive a product formula, which we use iteratively (iv) to prove the Trotter decomposition. Finally, (v) we show how the second claim of the theorem concerning the approximation with the average Liouvillian can be proven. Throughout the proof we consider times $t \geq s \geq 0$.

(i) Because any CPT map $T$ maps density matrices to density matrices, we have $\|T\|_{1 \rightarrow 1} \geq 1$. In Ref. [14] it is shown that

$$
\|T\|_{1 \rightarrow 1}=\sup _{A=A^{\dagger},\|A\|_{1}=1}\|T(A)\|_{1}
$$

for any CPT map $T$. Any self-adjoint operator $A=A_{+}-$ $A_{-}$can, by virtue of its spectral decomposition, be written as the difference of a positive and negative part $A_{ \pm} \geq 0$. Since $T$ is CPT, $\left\|T\left(A_{ \pm}\right)\right\|_{1}=\operatorname{tr}\left(T\left(A_{ \pm}\right)\right)=\|A\|_{1}$, hence $\|T\|_{1 \rightarrow 1} \leq 1$, and finally $\|T\|_{1 \rightarrow 1}=1$.

(ii) For any Liouvillian $\mathcal{K}$ the propagator $T_{\mathcal{K}}(t, s)$ is invertible and the inverse $T_{\mathcal{K}}^{-}(t, s)=\left(T_{\mathcal{K}}(t, s)\right)^{-1}$ is the unique solution of

$$
\frac{d}{d t} T^{-}(t, s)=-T^{-}(t, s) \mathcal{K}_{t}, \quad T^{-}(s, s)=\mathrm{id} .
$$

From the representation of $T^{-}$as a reversely time-ordered exponential, the inequality

$$
\left\|T_{\mathcal{K}}^{-}(t, s)\right\|_{1 \rightarrow 1} \leq \exp \left(\int_{s}^{t}\left\|\mathcal{K}_{r}\right\|_{1 \rightarrow 1} d r\right)
$$

follows. This can be proved rigorously with the ideas from Ref. [23] (see Supplemental Material [15]).

For the case where $\mathcal{K}$ is strictly $k$-local, we use its Lindblad representation and the inequality $\|A \rho B\|_{1} \leq$ $\|A\|_{\infty}\|\rho\|_{1}\|B\|_{\infty}$ to establish $\|\mathcal{K}\|_{1 \rightarrow 1} \in O\left(d^{k}\right)$ and hence $\left\|T_{\mathcal{K}}^{-}(t, s)\right\|_{1 \rightarrow 1} \leq e^{b(t-s)}$, with $b \in O\left(d^{k}\right)$. 
(iii) In the first step we use similar techniques as the ones being used for the unitary case [13] where differences of time evolution operators are bounded in operator norm by commutators of Hamiltonians. Applying the fundamental theorem of calculus twice, one can obtain for any two Liouvillians $\mathcal{K}$ and $\mathcal{L}$

$$
\begin{aligned}
T_{\mathcal{K}+\mathcal{L}}(t, s)-T_{\mathcal{K}}(t, s) T_{\mathcal{L}}(t, s) \\
=T_{\mathcal{K}}(t, s) T_{\mathcal{L}}(t, s) \int_{s}^{t} T_{\mathcal{L}}^{-}(r, s) \int_{s}^{r} \frac{d}{d u}\left(T_{\mathcal{K}}^{-}(u, s)\right. \\
\left.\quad \times \mathcal{L}_{r} T_{\mathcal{K}}(u, s)\right) T_{\mathcal{K}}^{-}(r, s) T_{\mathcal{K}+\mathcal{L}}(r, s) d u d r \\
=\int_{s}^{t} \int_{s}^{r} T_{\mathcal{K}}(t, s) T_{\mathcal{L}}(t, r) T_{\mathcal{K}}^{-}(u, s) \\
\quad \times\left[\mathcal{K}_{u}, \mathcal{L}_{r}\right] T_{\mathcal{K}}^{-}(r, u) T_{\mathcal{K}+\mathcal{L}}(r, s) d u d r .
\end{aligned}
$$

In the next step we take the $(1 \rightarrow 1)$-norm of this equation, use the triangle inequality, employ submultiplicativity of the norm, and use (i) and (ii) to obtain $\int_{s}^{t} \int_{s}^{r}\left\|\left[\mathcal{K}_{u}, \mathcal{L}_{r}\right]\right\|_{1 \rightarrow 1} d u d r$ as an upper bound. In the case where $\mathcal{K}$ and $\mathcal{L}$ are strictly $k$-local $\left\|\left[\mathcal{K}_{u}, \mathcal{L}_{r}\right]\right\|_{1 \rightarrow 1} \in$ $O\left(d^{2 k}\right)$, which follows by the same arguments used in (ii) to bound $\|\mathcal{K}\|_{1 \rightarrow 1}$. In the case where $\mathcal{L}$ is only $k$-local with $K$ terms, $\left\|\left[\mathcal{K}_{u}, \mathcal{L}_{r}\right]\right\|_{1 \rightarrow 1}$ is increased by at most the factor $K$ such that

$\left\|T_{\mathcal{K}+\mathcal{L}}(t, s)-T_{\mathcal{K}}(t, s) T_{\mathcal{L}}(t, s)\right\|_{1 \rightarrow 1} \in O\left((t-s)^{2} e^{b(t-s)} d^{2 k} K\right)$.

(iv) The propagator can be written as

$$
T_{\mathcal{L}}(\tau, 0)=\prod_{j=1}^{m} T_{\mathcal{L}}(\tau j / m, \tau(j-1) / m) .
$$

Using the inequality

$$
\left\|T_{1} T_{2}-\tilde{T}_{1} \tilde{T}_{2}\right\| \leq\left\|T_{1}\right\|\left\|T_{2}-\tilde{T}_{2}\right\|+\left\|T_{1}-\tilde{T}_{1}\right\|\left\|\tilde{T}_{2}\right\|
$$

and Eq. (14) iteratively, one can establish the result as stated in Eq. (8).

(v) For any strictly $k$-local Liouvillian $\mathcal{K}$ the propagator $T_{\mathcal{K}}(t, s)$ can be approximated by the propagator of the average Liouvillian,

$$
\left\|T_{\mathcal{K}}(t, s)-\exp \left(\int_{s}^{t} \mathcal{K}_{r} d r\right)\right\|_{1 \rightarrow 1}=\frac{1}{3} b(t-s)^{2} .
$$

This can be shown using the techniques described above by lifting the proof from Ref. [19] to the dissipative case (see Supplemental Material [15]). A comparison of Eq. (17) with Eq. (14) shows that the error introduced by using the average Liouvillian is small compared to the error introduced by the product decomposition and does not change the scaling of the error.

Conclusion.-In this work we show that under reasonable assumptions the dynamics of open quantum systems can be simulated efficiently by a circuit of local quantum channels in a Trotter-like decomposition. This channel circuit can further be simulated by a unitary quantum circuit with polynomially many gates from an arbitrary universal gate set. As a corollary it follows that the dissipative model of quantum computation is no more powerful than the standard unitary circuit model. Furthermore, the result implies that $k$-local Liouvillian dynamics can be simulated efficiently in the system size on a classical computer. It also shows that systems considered in the context of dissipative phase transitions [11,24] can be simulated in both of the above senses. The result can be seen as a quantum Church-Turing theorem in the sense that under reasonable and necessary requirements any general time evolution of an open quantum system can be simulated efficiently on a quantum computer.

This work was supported by the EU (Qessence, Minos, COQUIT, Compas), the BMBF (QuOReP), the EURYI, the Niels Bohr International Academy, the German National Academic Foundation, and the Perimeter Institute. We would like to thank M.P. Müller and T. Prosen for discussions.

[1] A. M. Turing, Proc. London Math. Soc. 42, 230 (1937); A. Church, Ann. Math. 33, 346 (1932).

[2] E. Bernstein, and U. Vazirani, SIAM J. Comput. 26, 1411 (1997).

[3] P. Kaye, R. Laflamme, and M. Mosca, An Introduction to Quantum Computing (Oxford University Press, Oxford, 2007).

[4] The Undecidable, Basic Papers on Undecidable Propositions, Unsolvable Problems And Computable Functions, edited by M. Davis (Raven Press, New York, 1965).

[5] R. Feynman, Int. J. Theor. Phys. 21, 467 (1982).

[6] S. Lloyd, Science 273, 1073 (1996).

[7] D. Aharonov, W. van Dam, J. Kempe, Z. Landau, S. Lloyd, and O. Regev, SIAM J. Comput. 37, 166 (2007); D. W. Berry, G. Ahokas, R. Cleve, and B. C. Sanders, Commun. Math. Phys. 270, 359 (2007).

[8] T. S. Cubitt, J. Eisert, and M. M. Wolf, arXiv:0908.2128.

[9] For finite dimensional systems this means mathematically that $T(t, s)$ defined in Eq. (5) satisfies (i) $T(t, r) T(r, s)=$ $T(t, s) \forall t \geq r \geq s \geq 0$ and (ii) $\lim _{\epsilon \rightarrow 0} \| T(t+\epsilon, t)-$ $\mathrm{id} \|=0 \forall t \geq 0$. Then $T$ is generated by a time dependent Liouvillian $\mathcal{L}_{t}$ [10].

[10] M. M. Wolf and J. I. Cirac, Commun. Math. Phys. 279, 147 (2008).

[11] F. Verstraete, M. M. Wolf, and J. I. Cirac, Nature Phys. 5, 633 (2009).

[12] G. Lindblad, Commun. Math. Phys. 48, 119 (1976).

[13] J. Huyghebaert and H. De Raedt, J. Phys. A 23, 5777 (1990).

[14] J. Watrous, Quantum Inf. Comput. 5, 58 (2005).

[15] See Supplemental Material at http://link.aps.org/ supplemental/10.1103/PhysRevLett.107.120501 for further details on the presented proofs and details on Implication 2. 
[16] W. F. Stinespring, Proc. Am. Math. Soc. 6, 211 (1955); V. Paulsen, Completely Bounded Maps and Operator Algebras (Cambridge University Press, Cambridge, England, 2002).

[17] C. M. Dawson and M. A. Nielsen, Quantum Inf. Comput. 6, 81 (2006); A. Yu. Kitaev, A. H. Shen, and M. N. Vyalyi, Classical and Quantum Computation (American Mathematical Society, Providence, RI, 2002), p. 257.

[18] A. Imamoglu, Phys. Rev. A 50, 3650 (1994); H. P. Breuer, Phys. Rev. A 70, 012106 (2004).

[19] D. Poulin, A. Qarry, R. D. Somma, and F. Verstraete, Phys. Rev. Lett. 106, 170501 (2011).

[20] We call states efficiently evaluable if they allow for an efficient evaluation of the expectation values of all strictly $k^{\prime}$-local observables for a given $k^{\prime} \in O(1)$. The stated implication follows from the fact that the application of
Trotter circuits to such observables yields again strictly $k^{\prime \prime}$-local observables with $k^{\prime \prime} \in O(1)$ due to a light cone effect.

[21] L. L. Halcomb and D. J. Diestler, J. Chem. Phys. 121, 3393 (2004); R. Kapral, Annu. Rev. Phys. Chem. 57, 129 (2006); D. M. Kernan, G. Ciccotti, and R. Kapral, J. Phys. Chem. B 112, 424 (2008); G. Benenti, G. Casati, T. Prosen, D. Rossini, and M. Znidaric, Phys. Rev. B 80, 035110 (2009).

[22] J. Barreiro et al., Nature (London) 470, 486 (2011).

[23] J. D. Dollard and C. N. Friedman, J. Math. Phys. (N.Y.) 18, 1598 (1977).

[24] S. Diehl, A. Micheli, A. Kantian, B. Kraus, H. P. Büchler, and P. Zoller, Nature Phys. 4, 878 (2008); J. Eisert and T. Prosen, arXiv:1012.5013. 